\title{
Unsteady forces on an accelerating plate and application to hovering insect flight
}

\author{
By D. I. PULLIN ${ }^{1}$ AND Z. JANE WANG \\ ${ }^{1}$ Graduate Aeronautical Laboratories, 105-50, California Institute of Technology, \\ Pasadena, CA 91125, USA \\ ${ }^{2}$ Theoretical and Applied Mechanics, Cornell University, Ithaca, NY 14853, USA
}

(Received 7 August 2003 and in revised form 10 November 2003)

The aerodynamic forces on a flat plate accelerating from rest at fixed incidence in two-dimensional power-law flow are studied analytically and numerically. An inviscid approximation is made in which separation at the two plate edges is modelled by growing spiral vortex sheets, whose evolution is determined by the BirkhoffRott equation. A solution based on a similarity expansion is developed, valid when the scale of the separated vortex is much smaller than the plate dimension. The leading order is given by the well-known similarity growth of a vortex sheet from a semi-infinite flat plate, while equations at the second order describe the asymmetric sweeping effect of that component of the free-stream parallel to the plate. Owing to subtle cancellation, the unsteady vortex force exerted on the plate during the starting motion is independent of the sweeping effect and is determined by the similarity solution, to the order calculated. This gives a mechanism for dynamic stall based on a combination of unsteady vortex lift and pure added mass; the incidence angle for maximum vortex lift is $\arccos \sqrt{3 / 8} \approx 52.2^{\circ}$ independent of the acceleration profile. Circulation on the flat plate makes no direct contribution. Both lift and drag force predictions from the unsteady inviscid theory are compared with those obtained from numerical solutions of the two-dimensional unsteady Navier-Stokes equations for an ellipse of high aspect ratio, and with predictions of Wagner's classical theory. There is good agreement with numerical results at high incidence and moderate Reynolds number. The force per unit span predicted by the vortex theory is evaluated for parameters typical of insect wings and is found to be in reasonable agreement with numerical simulations. Estimates for the shed circulation and the size of the start-up vortices are also obtained. The significance of this flow as a mechanism for insect hovering flight is discussed.

\section{Introduction}

The aerodynamics of insect flight have drawn fascination from both biologists and fluid dynamicists. See Weis-Fogh \& Jensen (1956), Maxworthy (1981), Ellington (1984), Dickinson (1996) for reviews. For hovering flight, various aerodynamic mechanisms have been proposed for lift generation by the timewise periodic motion of flapping wings (Weis-Fogh 1973; Lighthill 1977; Ellington 1984; Ellington et al. 1996; Dickinson, Lehmann \& Sane 1999; Wang 2000a). An understanding of this process can be viewed from two perspectives. The first refers to the near-wing vortex dynamics of producing local pressure lift in single wing strokes. Or equivalently, in 
order to maintain lift over many wing cycles, an insect must create a flux of downward momentum in the direction of the gravity vector.

In terms of near-wing aerodynamics, it is clear that unsteady vortex separation during the thrust stroke of the wing motion plays a crucial role in the production of forces on the heaving insect wing. This has been studied by direct numerical simulation of the Navier-Stokes equations in both two and three spatial dimensions; see Wang (2000a) and Sun \& Tang (2002). Wang (2000a) conducted numerical simulations, using a fourth-order finite difference scheme, of an elliptical wing undergoing a periodic heaving and pitching motion in a viscous fluid at Reynolds numbers of order $10-10^{3}$. The wing motion was strictly two-dimensional but nevertheless appeared to capture the essential features of the period-averaged force production via the generation of a pair of unsteady vortices during the downward stroke, and, crucially, the combination of the pair into a vortex dipole under the wing on the return stroke. This sequential pair/dipole production, coupled with the dipole's self-convection downward and away from the wing, then produces induced momentum flux and a subsequent reaction force on the body in a sense that can be qualitatively described by a momentum balance applied to the reverse von-Kármán vortex street: see Milne-Thomson (1968) and Saffman (1992). In three dimensions the dipoles will probably be replaced by arrays of possibly interconnected vortex-ring-like structures. See also Rayner(1979a,b) for a theoretical calculation of induced power assuming the wake to be a stack of coaxial vortex rings, Ellington (1984) for related models of vortex wakes and Freymuth et al. (1991) for experimental flow visualization of the wake.

Theoretical analysis of force generation during the translating phase of a flapping wing has been primarily based on Kutta-Joukowski's theory of a wing moving at a constant velocity, which ignores the influence of the near-wing vorticity and the effect of acceleration. Moreover, the theories of Wagner (1925) (linear) and of von Kármán \& Sears (1938) (nonlinear) include only the trailing-edge vortices and, as a result, are applicable to small angle of attack. At large angles of attack, as in the case of hovering insects, the separated vortices are better modelled by spiral vortex sheets, which assume a self-similar form at small times (Smith 1966; Pullin 1978; Saffman 1992). This idea was used by Graham (1983) to estimate the initial development of the lift on an aerofoil in inviscid starting flow producedby vortex separation at the trailing edge. He found singular behaviour at $t=0$ for the impulsive case and also argued that corrections produced by the non-singular part of the velocity field near the separating edge could have significant influence at finite times. Recent work by Jones (2003) formulated the separated flow around a moving plate using a regularized boundary-integral representation and obtained numerical solutions at angles of attack close to $90^{\circ}$.

In the present paper we use a conformal map and extend the similarity approach to model the unsteady vortex force produced during an accelerating phase of an insect wing stroke over the full range of angle of attack. The wing is modelled in two dimensions by a flat plate at incidence in inviscid starting flow. Separation from both the leading and trailing edges is modelled by spiral vortex sheets whose timewise evolution is asymmetrical about the normal to the wing centreline. The observation of Wang (2000a), that the vortex pair produced on the prior stroke quickly convects away from the wing, suggests that this localized model may be sufficient to analyse the vortex dynamics of lift generation.

In $\S 2$, the inviscid vortex dynamics of separation from a flat-plate aerofoil of finite chord is discussed and a similarity expansion of the integro-differential equation governing growth of the edge vortices is developed. It is shown in $\S 3$ that, to second 


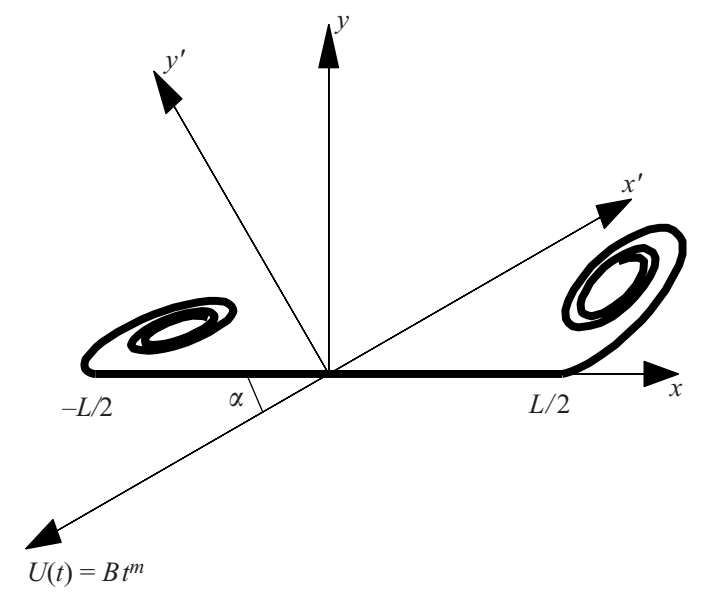

FIGURE 1. Definition of the plate motion and the two coordinate systems employed.

order, the effect of the finite part of the attached-flow velocity at the plate edges produces asymmetry in the growth of vortices from the leading and trailing wing edges but generates equal and opposite contributions to forces exerted on the wing, with subsequent cancellation. For small times, lift and drag forces on the wing are therefore dominated by the self-similar vortex growth. Circulation on the wing does not contribute. In $\S 4$ comparisons are made between the inviscid vortex theory, the classical theory of Wagner (1925), and numerical solutions of the Navier-Stokes equations for starting flow about an elliptical wing of high aspect ratio. Application of the vortex theory to force generation in simplified models of insect hovering flight motion is described in $\S 5$. The implications of the current theory for the aerodynamic basis of insect flight is discussed in $\S 6$.

\section{Flat plate in inviscid starting flow}

\subsection{Unsteady force on moving plate}

We consider two-dimensional starting flow about a flat plate of length $L$. This will represent a model of part of an insect wing motion that is sufficiently simple to be analytically tractable, yet which contains some of the essential dynamics of the unsteady vortex-lift generation process. In Cartesian coordinates $(x, y)$, in a laboratory-fixed frame of reference at time $t=0$, the plate is in $y=0,-L / 2 \leqslant x \leqslant L / 2$ (see figure 1). For $t \geqslant 0$, the plate moves into the third quadrant, without rotation, with speed $U(t)$ and in a straight-line trajectory such that its centreline makes an angle $\alpha$ with the negative $x$-axis. The plate speed profile is of power-law form

$$
U(t)=B t^{m},
$$

where $m \geqslant 0$ is a power exponent and $B$ constant with dimensions Length $\times$ Time $^{-(1+m)}$. This kinematic is chosen for analytical simplicity in describing the unsteady vortex dynamics of separation at the plate edges; it can be thought of as a single-stroke wing motion. We wish to determine the time-dependent force on the plate generated by the start-up flow for $t \geqslant 0$. It is assumed that the flow is twodimensional and inviscid but the effect of viscosity $v$ in the limit $v \rightarrow 0$ is modelled by allowing separation at the plate edges $x= \pm L / 2$ in the form of a pair of unsteady vortex sheets. These roll up into structures to be specified subsequently. The flow is 
considered to be everywhere irrotational, save for the singular distribution of vorticity along the vortex sheets.

It is convenient to work in a non-inertial frame of reference at rest with respect to the plate. In this frame let $\mathscr{C}(t)$ be a closed contour surrounding and immediately adjacent to the plate and both vortex sheets. We now introduce the complex potential

$$
W(z, t)=\phi(x, y, t)+\mathrm{i} \psi(x, y, t),
$$

where $z=x+\mathrm{i} y, \phi$ is the velocity potential and $\psi$ is the stream function. The complex velocity is $u(x, y, t)-\mathrm{i} v(x, y, t)=\mathrm{d} W / \mathrm{d} z$ and, at $z \rightarrow \infty$, the complex velocity relative to the plate is then $U(t)-\mathrm{i} V(t)=U(t) \exp (-\mathrm{i} \alpha)$. Since the total circulation around the plate-vortex system is always zero, the instantaneous force per unit length exerted by the fluid on the plate can be expressed as (Newman 1977; Milne-Thomson 1968)

$$
\begin{aligned}
F_{y}-\mathrm{i} F_{x} & =-\rho \frac{\mathrm{d}}{\mathrm{d} t} \oint_{\mathscr{C}(t)}\left[W(z, t)-W_{\infty}(z, t)\right] \mathrm{d} z, \\
W_{\infty}(z, t) & =U(t) \exp (-\mathrm{i} \alpha) z,
\end{aligned}
$$

where $\rho$ is the fluid density, $F_{x}, F_{y}$ are the components of the force per unit span on the plate in the $x$ - and $y$-directions respectively and the integration is anti-clockwise on the contour $\mathscr{C}(t) . W(z, t)$ must be such that, $\mathrm{d}\left[W(z, t)-W_{\infty}(z, t)\right] / \mathrm{d} z \sim O\left(z^{-2}\right)$, $z \rightarrow \infty$. Under these conditions, an integral on the right-hand side of (2.3) giving the momentum flux across, and the external pressure force on a large circle at infinity can be shown to vanish.

\subsection{Vortex-sheet model of edge separation}

To construct $W(z, t)$ we first introduce the conformal mapping

$$
\zeta(z)=\frac{1}{2}\left(z+\sqrt{z^{2}-L^{2} / 4}\right),
$$

that maps the $z$-plane exterior to the plate to the exterior of the circle $|\zeta|=L / 4$ in the $\zeta$-plane. The complex potential can now be written as

$$
W(z, t)=U(t) w_{a}(z)+W_{v}(\zeta(z), t)
$$

where

$$
w_{a}(z)=z \cos \alpha-\mathrm{i} \sin \alpha \sqrt{z^{2}-L^{2} / 4}
$$

gives the attached flow and $W_{v}(z, t)$ is the complex potential produced by separation at the plate edges. The attached flow satisfies the boundary condition $\operatorname{Im}\left[w_{a}\right]=0$, on $y=0,-L / 2<x<L / 2$ as it should, where Im denotes the imaginary part.

To construct the complex potential for the separated flow we introduce two free vortex sheets, each of which emanates from one of the two sharp edges at $z=L / 2$, $z=-L / 2$. Each vortex sheet, denoted by the complex functions $Z_{+}(\Gamma, t)$ and $Z_{-}(\Gamma, t)$ respectively, is paramaterized by $\Gamma$, where $\Gamma$ is a Lagrangian parameter for each sheet that lies in the range $0<|\Gamma|<\left|\Gamma_{ \pm}(t)\right|$. This is a standard representation of vortex sheets well-suited to a compact description of their dynamical evolution; see Saffman (1992). The parameter $\Gamma$ is the total circulation concentrated on the sheet between a point on the sheet and the sheet tip, and $\Gamma_{ \pm}(t)$ is the total shed circulation at time $t$ on sheet \pm . The functions $Z_{+}(\Gamma, t)$ and $Z_{-}(\Gamma, t)$ satisfy the Euler dynamics of vortex-sheet evolution for which $\Gamma$ is a conserved quantity moving with the average velocity of two instantaneously adjacent fluid particles, one on each side of the sheet. 
Now let

$$
\mathscr{Z}_{ \pm}=\frac{1}{2}\left(Z_{ \pm}+\sqrt{Z_{ \pm}^{2}-L^{2} / 4}\right)
$$

where $\mathscr{Z}_{ \pm}=\mathscr{Z}_{ \pm}(\Gamma, t)$ are the vortex sheets mapped onto the $\zeta$-plane. The complex potential contributed by the separated vortex sheets at time $t$ can then be written as

$$
W_{v}(\zeta, t)=\frac{1}{2 \pi \mathrm{i}} \sum_{ \pm} \int_{0}^{\Gamma_{ \pm}(t)}\left[\log \left(\zeta-\mathscr{Z}_{ \pm}(\Gamma, t)\right)-\log \left(\zeta-\frac{L^{2}}{16 \mathscr{Z}_{ \pm}^{*}(\Gamma, t)}\right)\right] \mathrm{d} \Gamma
$$

where the sum, used here and elsewhere, is over the \pm sheets, * denotes the complex conjugate and where images of the sheets in the circle $|\zeta|=L / 4$ have been used to satisfy the boundary condition for $W_{v}$, namely that $\psi_{v}=\operatorname{Im}\left[W_{v}\right]$ is constant on the plate surface in the $z$-plane.

The force on the moving plate can now be obtained by substituting (2.7) and (2.9) together with (2.5) into (2.6) and then into (2.3). For both the components contributed by the attached and separated flow, the resulting integrals can be readily evaluated by expanding $\mathscr{C}(t)$ to infinity and applying the method of residues. The attached flow $w_{a}$ then gives the well-known result

$$
F_{y}^{(a)}=\rho L^{2} \sin \alpha \frac{\pi}{4} \frac{\mathrm{d} U(t)}{\mathrm{d} t} .
$$

This is just the force produced by the attached-flow added mass accelerating at $\mathrm{d} U(t) / \mathrm{d} t$. For $W_{v}$, the contour integral is most easily handled in the $\zeta$-plane, and it is found that the additional force per unit length on the plate produced by the separated flow is then

$$
F_{y}^{(v)}-\mathrm{i} F_{x}^{(v)}=-\rho \frac{\mathrm{d}}{\mathrm{d} t} \sum_{ \pm} \int_{0}^{\Gamma_{ \pm}(t)}\left(\frac{L^{2}}{16 \mathscr{Z}_{ \pm}^{*}(\Gamma, t)}-\mathscr{Z}_{ \pm}(\Gamma, t)\right) \mathrm{d} \Gamma .
$$

We shall refer to this as the vortex force.

\section{Vortex dynamics}

\subsection{Birkhoff-Rott equation}

Evaluation of (2.11) requires a solution for the dynamical evolution $\mathscr{Z}_{ \pm}(\Gamma, t)$, or equivalently $Z_{ \pm}(\Gamma, t)$, for given $U(t)$. This can be obtained from the Birkhoff-Rott equation (Rott 1956) for the vortex sheet growing from $z=(L / 2,0)$

$$
\frac{\partial Z_{+}^{*}(\Gamma, t)}{\partial t}=\frac{\mathrm{d} W}{\mathrm{~d} z}\left(z=Z_{+}(\Gamma, t)\right) .
$$

Using (2.5)-(2.9), this can be written as

$$
\begin{aligned}
\frac{\partial Z_{+}^{*}(\Gamma, t)}{\partial t}=B t^{m} & \left(\cos \alpha-\frac{\mathrm{i} Z_{+} \sin \alpha}{\sqrt{Z_{+}^{2}-L^{2} / 4}}\right)+\frac{1}{2}\left(1+\frac{Z_{+}}{\sqrt{Z_{+}^{2}-L^{2} / 4}}\right) \\
& \times \frac{1}{2 \pi \mathrm{i}} \int_{0}^{\Gamma(t)}\left(\frac{1}{\mathscr{Z}_{+}-\mathscr{Z}_{+}^{\prime}}-\frac{1}{\mathscr{Z}_{+}-L^{2} /\left(16 \mathscr{Z}_{+}^{* \prime}\right)}\right) \mathrm{d} \Gamma^{\prime},
\end{aligned}
$$

where $Z_{+} \equiv Z_{+}(\Gamma, t)$ and $\mathscr{Z}^{\prime}{ }_{+} \equiv \mathscr{Z}_{+}\left(\Gamma^{\prime}, t\right)$. In (3.2) we have omitted the velocity induced on the vortex near $z=L / 2$ by the vortex shed from $z=-L / 2$. In the expansion that follows, this term can be shown to be of higher formal order, for sufficiently small 
$t$, than the order-one attached-flow velocity near $z=L / 2$. Equation (3.2) is a highly nonlinear problem of vortex dynamics that will in general require numerical treatment. Presently we make use of an analytical approximation in the form of a similarity expansion that, for finite plate length $L$, will provide an accurate description of the vortex dynamics only for times sufficiently small such that $\left|Z_{+}(\Gamma, t)-L / 2\right| / L \ll 1$. Although this condition will be seen to be violated at late times in application to insect wing aerodynamics, the resulting force may nevertheless be expected to provide a reasonable analytical estimate of the unsteady vortex force in terms of parameters that characterize hovering flight.

\subsection{Similarity expansion}

The first term on the right-hand side of (3.2) is the complex velocity provided by the attached flow. If we put $\tilde{Z}_{+}=Z_{+}-L / 2$ and expand in powers of $\tilde{Z}_{+} / L \ll 1$ about $\tilde{Z}_{+}=0$, it is found that, to leading order, this term is singular and of the form

$$
\frac{\mathrm{d} W_{a}}{\mathrm{~d} z}\left(z=Z_{+}\right)=-\frac{\mathrm{i}}{2} a t^{m} \tilde{Z}_{+}^{-1 / 2}+\cdots,
$$

where

$$
a=L^{1 / 2} B \sin \alpha .
$$

Equation (3.3) gives the attached flow past a semi-infinite flat plate for which there is no natural, fixed length scale. This can be used to construct a leading-order similarity solution to (3.2), valid for sufficiently small $t$ (Smith 1966; Pullin 1978), for which the appropiate time-dependent length scale is

$$
\begin{aligned}
\delta(t) & =K a^{2 / 3} t^{2(1+m) / 3}, \\
K & =\left[\frac{3}{4(1+m)}\right]^{2 / 3},
\end{aligned}
$$

where $K$ is a convenient scaling constant.

The angle $\alpha$ affects the similarity sheet evolution for small $t$ only through the parameter $a$. Since the dominant term in the expansion of the attached-flow velocity is essentially the same near $z= \pm L / 2$, it follows that, at leading order, the vortex sheets shed from $z= \pm L / 2$ are symmetric about $x=0$; their circulations are equal and opposite, as are their image circulations on the plate. The plate circulation is therefore zero. In what follows, we wish to obtain asymmetric corrections to the leading-order solution and to evaluate the subsequent effect on the vortex force exerted on the plate. Motivated by (3.5), we thus introduce the similarity expansion

$$
\begin{aligned}
Z_{+}(\Gamma, t) & =\frac{L}{2}\left(1+2 \epsilon^{2} \omega_{0}(\lambda)+2 \epsilon^{2+\mu_{1}}(t) \omega_{1}(\lambda) \cdots\right), \\
\Gamma & =\frac{\delta^{2}(t) t^{-1}}{K^{3 / 2}}\left(J_{0}+\epsilon^{\mu_{2}}(t) J_{1}+\cdots\right)(1-\lambda),
\end{aligned}
$$

where $\epsilon=(\delta(t) / L)^{1 / 2} \ll 1$ is a time-dependent parameter and $\lambda$ is a dimensionless circulation parameter. In (3.7)-(3.8), $\omega_{0}(\lambda)$ and $\omega_{1}(\lambda)$ are complex shape functions, and $J_{0}$ and $J_{1}$ are dimensionless constants to be determined. It will be argued subsequently that the exponents $\mu_{1}$ and $\mu_{2}$ must take the values $\mu_{1}=\mu_{2}=1$, and these values will now be assumed. 
When (3.7) and (3.8) are substituted into (3.2), and an expansion in powers of $\epsilon(t)$ is made, after taking care with evaluation of the time derivative on the left-hand side at fixed $\Gamma$, it is found that all terms scale as $\delta(t) t^{-1} \epsilon^{p}(t), p=0,1,2, \ldots$ Cancelling $\delta(t) t^{-1}$ uniformly and equating terms of order $\epsilon^{0}$ then gives

$$
\begin{aligned}
\omega_{0}^{*}+Q(1-\lambda) \frac{\mathrm{d} \omega_{0}^{*}}{\mathrm{~d} \lambda} & =\frac{1}{\omega_{0}^{1 / 2}}\left(-\mathrm{i}+\frac{J_{0}}{2 \pi \mathrm{i}} \int_{0}^{1} F\left(\omega_{0} \mid \omega_{0}^{\prime}\right) \mathrm{d} \lambda^{\prime}\right), \\
F\left(\omega_{0} \mid \omega_{0}^{\prime}\right) & =\frac{1}{\omega_{0}^{1 / 2}-\omega_{0}^{\prime 1 / 2}}-\frac{1}{\omega_{0}^{1 / 2}+\omega_{0}^{* 1 / 2}},
\end{aligned}
$$

where $\omega_{0} \equiv \omega_{0}(\lambda), \omega_{0}^{\prime} \equiv \omega_{0}\left(\lambda^{\prime}\right)$ and $Q=(4 m+1) /(2 m+2)$. When $\lambda \rightarrow 0$, then $\omega_{0} \rightarrow 0$ and the right-hand side of (3.9) is unbounded unless the (zeroth-order) Kutta condition is satisfied:

$$
1+\frac{J_{0}}{2 \pi} \int_{0}^{1} F\left(0 \mid \omega_{0}\right) \mathrm{d} \lambda=0 .
$$

This condition determines $J_{0}$. Equation (3.9) is a nonlinear integral equation defining $\omega_{0}(\lambda)$. Its numerical solution (Pullin 1978) gives the similarity solution for starting flow past a semi-infinite flate plate discussed above.

At $O(\epsilon)$, it is found that

$$
\begin{array}{r}
\frac{3}{2} \omega_{1}^{*}+Q(1-\lambda) \frac{\mathrm{d} \omega_{1}^{*}}{\mathrm{~d} \lambda}+\frac{J_{1}}{2 J_{0}}(1-\lambda) \frac{\mathrm{d} \omega_{0}^{*}}{\mathrm{~d} \lambda}=2 \cot \alpha+\frac{1}{2} \frac{\omega_{1}}{\omega_{0}^{3 / 2}}\left(\mathrm{i}-\frac{J_{0}}{2 \pi \mathrm{i}} \int_{0}^{1} F\left(\omega_{0} \mid \omega_{0}^{\prime}\right) \mathrm{d} \lambda^{\prime}\right) \\
-\frac{1}{\omega_{0}^{1 / 2}} \frac{J_{0}}{2 \pi \mathrm{i}} \int_{0}^{1} G\left(\omega_{0}, \omega_{1} \mid \omega_{0}^{\prime}, \omega_{1}^{\prime}\right) \mathrm{d} \lambda^{\prime}+\frac{1}{\omega_{0}^{1 / 2}} \frac{J_{1}}{2 \pi \mathrm{i}} \int_{0}^{1} F\left(\omega_{0} \mid \omega_{0}^{\prime}\right) \mathrm{d} \lambda^{\prime}, \quad(3.12)
\end{array}
$$

where

$$
2 G\left(\omega_{0}, \omega_{1} \mid \omega_{0}^{\prime}, \omega_{1}^{\prime}\right)=\frac{\omega_{1} \omega_{0}^{\prime 1 / 2}-\omega_{1}^{\prime} \omega_{0}^{1 / 2}}{\omega_{0}^{1 / 2} \omega_{0}^{\prime 1 / 2}\left(\omega_{0}^{1 / 2}-\omega_{0}^{\prime 1 / 2}\right)^{2}}-\frac{\omega_{1} \omega_{0}^{*^{\prime 1}}{ }^{1 / 2}+\omega^{* \prime} \omega_{0}{ }^{1 / 2}}{\omega_{0}^{1 / 2} \omega_{0}^{*^{1 / 2}}\left(\omega_{0}^{1 / 2}+\omega_{0}^{* 1 / 2}\right)^{2}} .
$$

Equation (3.12) is a singular but linear integral equation for $\omega_{1}(\lambda)$. This gives a correction to the similarity solution defined by (3.9) and can be solved only when $\omega_{0}(\lambda)$ and $J_{0}$ are known. When $\lambda \rightarrow 0$, then $\omega_{0} \rightarrow 0$ and $\omega_{1} \rightarrow 0$ (this follows from (3.7)) and terms on the right-hand side contain singular factors. In this limit, using (3.9), the terms multiplied by $\omega_{0}^{-3 / 2}$ may be shown to be bounded. Using (3.11), it follows that the remaining terms on the right-hand side are bounded if the $O(\epsilon)$ Kutta condition

$$
J_{1}-\frac{J_{0}^{2}}{4 \pi} \int_{0}^{1}\left(\frac{\omega_{1}}{\omega_{0}^{3 / 2}}+\frac{\omega^{*}{ }_{1}}{\omega^{* 3 / 2}}\right) \mathrm{d} \lambda=0
$$

is satisfied. This is sufficient to determine $J_{1}$, which gives a correction to the vortex circulation predicted by the similarity solution. We remark that, unless $\mu_{1}=\mu_{2}=1$, it is not possible to relieve all singular terms in the expansion of (3.2) when $\lambda \rightarrow 0$, uniformly in time. Putting $\lambda=0$ in (3.8) gives the circulation shed at time $t$

$$
\Gamma_{+}(t)=K^{1 / 2} a^{4 / 3} t^{4(1+m) / 3-1}\left(J_{0}+J_{1} K^{1 / 2} L^{-1 / 2} a^{1 / 3} t^{(1+m) / 3}+\cdots\right) .
$$

From (3.7) with $\lambda=1$ we obtain the locus of the vortex centre

$$
\tilde{Z}_{+}(0, t)=K a^{2 / 3} t^{2(1+m) / 3}\left(\omega_{0}(1)+K^{1 / 2} L^{-1 / 2} a^{1 / 3} t^{(1+m) / 3} \omega_{1}(1)+\cdots\right) .
$$


Equation (3.12) describes the effect of asymmetry in the vortex-sheet evolution from the edge $z=L / 2$. This is driven by the first term on the right-hand side, which is produced by the 'sweeping' component of the fluid velocity at infinity tangential to the plate. The $\alpha$-dependence can be removed explicitly from (3.12) by rescaling, from which it follows that both $J_{1}$ and $\omega_{1}$ are proportional to $\cot \alpha$. Although a similar equation for the sheet shed at $z=-L / 2$ can be obtained, this case can be deduced using symmetry arguments applied to (3.12) as follows: for $m \geqslant 0$ and $\alpha<\pi / 2$ let $\omega_{0}(\lambda)$ and $J_{0}, \omega_{1}(\lambda)$ and $J_{1}$ be solutions to (3.9)-(3.11) and to (3.12)-(3.14) respectively, representing the leading-order and sweeping-asymmetry correction for the sheet shed at $z=L / 2$. Because $\alpha<\pi / 2$ (see figure 1), we denote this $(+$ ) sheet a leeward separation, and that $(-)$ from $z=-L / 2$ a windward separation. The effect of the sweeping asymmetry on the windward sheet can be inferred by replacing $\alpha$ by $\pi-\alpha$ in (3.12)-(3.14). At fixed time $t$, we note that this has the effect of reflecting the whole asymmetric vortex structure about the normal to the plate centreline ( $y$-axis), so that the sheet from $z=L / 2$ is then 'windward' while that from $z=-L / 2$ becomes 'leeward'. But when $\alpha \rightarrow \pi-\alpha$, it can be seen that (3.12)-(3.14) are invariant under $\omega_{1} \rightarrow-\omega_{1}$ and $J_{1}=\rightarrow-J_{1}$. Applying this result to the sheet from $z=-L / 2$, and taking into account the reflection property discussed above, it follows that, when $\alpha<\pi / 2$, the circulation and shape of this sheet are respectively

$$
\begin{aligned}
\Gamma_{-}(t) & =K^{1 / 2} a^{4 / 3} t^{4(1+m) / 3-1}\left(-J_{0}+J_{1} K^{1 / 2} L^{-1 / 2} a^{1 / 3} t^{(1+m) / 3}+\cdots\right), \\
Z_{-}(\Gamma, t)+\frac{L}{2} & =K a^{2 / 3} t^{2(1+m) / 3}\left(-\omega_{0}^{*}(\lambda)+K^{1 / 2} L^{-1 / 2} a^{1 / 3} t^{(1+m) / 3} \omega_{1}^{*}(\lambda)+\cdots\right) .
\end{aligned}
$$

This result can also be obtained by the simple physical argument that, at fixed $\alpha$, the sweeping flow is equal and opposite at the leading/trailing edges of the plate. The dimensionless circulation on the plate produced by sweeping asymmetry is equal to $2 J_{1}$.

\subsection{Point-vortex model}

The effects of asymmetry can be evaluated explicitly by the use of a point-vortex model. Here, for simplicity, the entire vortex sheet is concentrated into a single point vortex joined by a cut in the $z$-plane to $z=L / 2$. The point-vortex equations can be obtained by first replacing the Birkhoff-Rott equation by the requirement that the force on the vortex-cut system be zero as the vortex increases in circulation and moves away from $z=L / 2$. This is then followed by the application of a similarity expansion analogous to (3.7)-(3.8) to the result. An alternative is to integrate (3.9)-(3.14) in $0 \leqslant \lambda \leqslant 1$ and to identify the right-hand side with point-vortex kinematics. In either case care must be exercised in evaluating the point-vortex self-induction using Routh's theorem (Milne-Thomson 1968). The results obtained using both methods agree, with the result that the point-vortex similarity-expansion equations are, to leading order,

$$
\begin{aligned}
(1+Q) \omega_{0 v}^{*} & =-\frac{\mathrm{i}}{\omega_{0 v}^{1 / 2}}+\frac{J_{0}}{2 \pi \mathrm{i}}\left(-\frac{1}{2 \omega_{0 v}}+\frac{1}{\omega_{0 v}^{1 / 2}} F_{v}\left(\omega_{0 v}\right)\right), \\
1 & =\frac{J_{0}}{2 \pi}\left(\frac{1}{\omega_{0 v}^{1 / 2}}+\frac{1}{\omega_{0 v}^{* 1 / 2}}\right), \\
F_{v}\left(\omega_{0 v}\right) & =-\frac{1}{\omega_{0 v}^{1 / 2}+\omega_{0 v}^{* 1 / 2}},
\end{aligned}
$$

where $\omega_{0 v}$ is the leading-order position of the point vortex. The $O\left(\epsilon^{1}\right)$ equations are 


$$
\begin{array}{r}
\left(\frac{3}{2}+Q\right) \omega_{1 v}^{*}+\frac{J_{1}}{2 J_{0}} \omega_{0 v}^{*}=2 \cot \alpha-\frac{J_{1}}{4 \pi \mathrm{i} \omega_{0 v}}+\frac{J_{0} \omega_{1 v}}{4 \pi \mathrm{i} \omega_{0 v}^{2}}+\frac{1}{2} \frac{\omega_{1 v}}{\omega_{0 v}^{3 / 2}}\left(\mathrm{i}-\frac{J_{0}}{2 \pi \mathrm{i}} F_{v}\left(\omega_{0 v}\right)\right) \\
-\frac{1}{\omega_{0 v}^{1 / 2}}\left(\frac{J_{0}}{2 \pi \mathrm{i}} G_{v}\left(\omega_{0 v}, \omega_{1 v}\right)-\frac{J_{1}}{2 \pi \mathrm{i}} F_{v}\left(\omega_{0 v}\right)\right),
\end{array}
$$

with associated Kutta condition

$$
J_{1}=\frac{J_{0}^{2}}{4 \pi}\left(\frac{\omega_{1 v}}{\omega_{0 v}^{3 / 2}}+\frac{\omega^{*} 1 v}{\omega_{0 v}^{* 3 / 2}}\right)
$$

where

$$
2 G_{v}\left(\omega_{0 v}, \omega_{1 v}\right)=-\frac{\omega_{1 v} \omega_{0 v}^{* 1 / 2}+\omega_{1 v}^{*} \omega_{0 v}^{1 / 2}}{\omega_{0 v}^{1 / 2} \omega_{0 v}^{* 1 / 2}\left(\omega_{0 v}^{1 / 2}+\omega_{0 v}^{* 1 / 2}\right)^{2}},
$$

and $\omega_{1 v}$ is the $O\left(\epsilon^{1}\right)$ correction to the point-vortex position in the similarity expansion.

The point-vortex equations can be solved analytically. The solution to (3.19)-(3.21) is (Rott 1956)

$$
\omega_{0 v}=\frac{\mathrm{i}}{2^{1 / 3}}\left(\frac{1+m}{3+6 m}\right)^{2 / 3}, \quad J_{0}=2^{1 / 3} \pi\left(\frac{1+m}{3+6 m}\right)^{1 / 3} .
$$

When these results are substituted into (3.22)-(3.23), their solution can be obtained as

$$
\begin{aligned}
\omega_{1 v} & =\frac{4(1+m)}{7+13 m} \cot \alpha\left(1+\frac{\mathrm{i}}{3}\right), \\
J_{1} & =-4\left(\frac{2}{3}\right)^{2 / 3} \frac{(1+m)^{2 / 3}(1+2 m)^{1 / 3}}{7+13 m} \pi \cot \alpha .
\end{aligned}
$$

When $0<\alpha<\pi / 2$, the effect of asymmetry on the vortex from $z=L / 2$ is to weaken the (positive) vortex circulation and to cause it to move outboard and further above the plane of the plate in comparison to the trajectory of the leading-order similarity solution. The asymmetric effect on the vortex near $z=-L / 2$ is the opposite: the vortex circulation increases in magnitude (becomes more negative) and the vortex moves, relatively, inboard and towards the plate surface. Cortelezzi \& Leonard (1993) obtained a point-vortex solution for power-law starting flow past a semi-infinite flat plate in the presence of parallel plate motion, also of power-law form in time. They consider cases for which the power exponents of $t$ for these velocities are generally different. For an appropriate choice of matching parameters, (3.25)-(3.27) are in agreement with their equations (24).

\subsection{Vortex force exerted on plate}

The vortex force can now be evaluated by applying (3.7)-(3.8) to (2.11). First write this equation as

$$
F_{y}^{(v)}-\mathrm{i} F_{x}^{(v)}=-\rho \frac{\mathrm{d}}{\mathrm{d} t}\left(I_{+}(t)+I_{-}(t)\right),
$$


where $I_{+}(t), I_{-}(t)$ are the integrals in (2.11) contributed by the vortices shed at $z=L / 2$, $z=-L / 2$ respectively. Applying (3.7)-(3.8) to $I_{+}(t)$, gives, after some algebra

$$
\begin{aligned}
I_{+}(t)=-\frac{L^{1 / 2} \delta^{5 / 2}(t) t^{-1}}{2 K^{3 / 2}} & {\left[J_{0} \int_{0}^{1}\left(\omega_{0}^{1 / 2}+\omega_{0}^{* 1 / 2}\right) \mathrm{d} \lambda+\epsilon(t)\left(J_{1} \int_{0}^{1}\left(\omega_{0}^{1 / 2}+\omega_{0}^{* 1 / 2}\right) \mathrm{d} \lambda\right.\right.} \\
+ & \left.\left.J_{0} \int_{0}^{1}\left(\frac{\omega_{1}}{2 \omega_{0}^{1 / 2}}+\frac{\omega_{1}^{*}}{2 \omega_{0}^{* 1 / 2}}+\omega_{0}-\omega_{0}^{*}\right) \mathrm{d} \lambda\right)+O\left(\epsilon^{2}\right)\right] .
\end{aligned}
$$

A similar expression can be obtained for $I_{-}(t)$. When these results are used in (3.28) together with (3.17)-(3.18), it is found that the $O(\epsilon)$ contribution to the vortex force contributed by asymmetric corrections vanishes owing to cancellations. As a result, the vortex force can be expressed as

$$
F_{y}^{(v)}=2\left(\frac{5 m+2}{3}\right) K \rho L^{1 / 2} J_{0} a^{5 / 3} t^{5(1+m) / 3-2} \operatorname{Re}\left[\int_{0}^{1} \omega_{0}^{1 / 2}(\lambda) \mathrm{d} \lambda\right]+O\left(t^{7(m+1) / 3}\right),
$$

where the cancelling terms are each of $O\left(t^{2 m}\right)$. In (3.30), the leading-order power-law scaling in time agrees with Graham (1983). The present result, that the leading-order, asymmetric correction to the vortex force vanishes can be understood as follows: the relative movements of the separated vortex systems produced by asymmetry will tend to enhance vortex-induced, low-pressure suction under the windward vortex as it moves inboard near $z=-L / 2$, while relieving this effect near the leeward vortex, which moves outboard from $z=L / 2$. To the order calculated, these effects cancel. The presence of circulation on the plate, produced by asymmetry in the two shed vortices, plays no direct role in the mechanism of force production. The integral in (3.30) can be estimated either from the point-vortex solution (3.25) or from detailed similarity solutions for the vortex-sheet evolution. The latter show that $\omega_{0}(\lambda)$ is a function that oscillates rapidly about the centre of roll-up $\omega_{0}(1)$. Hence, for present purposes we approximate

$$
\operatorname{Re}\left[\int_{0}^{1} \omega_{0}^{1 / 2}(\lambda) \mathrm{d} \lambda\right] \approx \operatorname{Re}\left[\omega_{0}^{1 / 2}(\lambda=1)\right]
$$

The numbers $J_{0}$ and $\omega_{0}(1)$ used here are from Pullin (1978) but the point-vortex solution could also be used to provide an approximate analytical expression for the vortex force.

\section{Comparison with Navier-Stokes simulations and Wagner's theory}

We now compare the unsteady forces derived above to the Navier-Stokes simulations of an accelerating ellipse at angles of attack ranges from $0^{\circ}$ to $90^{\circ}$. In particular, we shall consider the linearly accelerating case, where $m=1$. For this purpose it is convenient to rotate axes by $\alpha$ in the counter-clockwise direction on to axes $\left(x^{\prime}, y^{\prime}\right)$, with rotation matrix $[R(\alpha)]$, such that $\left(x^{\prime}, y^{\prime}\right)^{T}=[R(\alpha)](x, y)^{T}$. In the $\left(x^{\prime}, y^{\prime}\right)$ system, the ellipse accelerates in the negative $x^{\prime}$-direction with velocity $U(t)$ and at angle of attack $\alpha$.

\subsection{Wagner theory}

In the case of small $\alpha$, it is also appropriate to compare our results with predictions from Wagner's classical theory of an unsteady airfoil (Wagner 1925). The main difference between Wagner's theory and the present approach is the assumption 
about the shed vortices. Wagner includes the vortex shed from the trailing edge only, which is a reasonable approximation at small angle of attack. In addition, Wagner's theory ignores the roll-up of the vortex sheet, but assumes that the vortex distribution shed at time $t$ is convected by $U(t)$, the velocity of the airfoil at time $t$. Despite these simplifications, Wagner's theory predicts the unsteady circulation around an accelerating airfoil remarkably well, as verified by the experiments of Walker (1931).

In the case of $m=1$, Wagner's theory predicts (von Kármán \& Burgers 1963; Childress 1981)

$$
\begin{aligned}
& F_{x^{\prime}}^{w}=\frac{\mathrm{d}}{\mathrm{d} t}\left(m_{11}^{\prime} U\right), \\
& F_{y^{\prime}}^{w}=\rho \pi L U^{2}(t) \sin \alpha+\frac{\mathrm{d}}{\mathrm{d} t}\left(m_{21}^{\prime} U(t)\right)+\frac{1}{2} \rho U(t) L \int_{0}^{X(t)} \frac{\gamma(\xi, t)}{\sqrt{L \xi+\xi^{2}}} \mathrm{~d} \xi,
\end{aligned}
$$

where $X(t)$ the distance travelled, $\gamma(\xi, t)$ is the circulation per unit length in the $x$ direction of the vortex sheet shed at the trailing edge, and $m_{11}^{\prime}, m_{21}^{\prime}$ are components of the added mass tensor $m_{i j}^{\prime}$. In the $x^{\prime}, y^{\prime}$ coordinates, $[m]=\left[R^{-1}(\alpha)\right]\left[m^{\prime}\right][R(\alpha)]$, where $[m]$ is the added mass tensor in the $x, y$ or body coordinates, and $[R(\alpha)]$ the rotation matrix between the two coordinate systems. At small time $(\xi / L \ll 1)$, the integral in (4.2) can be approximated by

$$
\begin{aligned}
L \int_{0}^{X(t)} \frac{\gamma(\xi, t)}{\sqrt{L \xi+\xi^{2}}} \mathrm{~d} \xi & \sim \int_{0}^{X(t)} \gamma(\xi, t) \sqrt{\frac{L+\xi}{\xi}} \mathrm{d} \xi \\
& \sim-\pi L U(t) \sin \alpha
\end{aligned}
$$

and therefore

$$
F_{y^{\prime}}^{w}=\frac{1}{2} \rho \pi L U^{2} \sin \alpha+\frac{\mathrm{d}}{\mathrm{d} t}\left(m_{21}^{\prime} U\right),
$$

where the first term is equal to half of the steady state value. The net force due to circulation is orthogonal to the relative flow at infinity.

In contrast, the vortex force predicted from the current theory is normal to the plate, and from (3.31) and using (3.4) with $m=1$ it is found that

$$
F_{n}=\frac{14}{3} \rho K J_{0} B^{5 / 3} L^{4 / 3} \sin ^{5 / 3} \alpha t^{4 / 3} \operatorname{Re}\left[\int_{0}^{1} \omega_{0}^{1 / 2}(\lambda) \mathrm{d} \lambda\right] .
$$

The vortex-sheet similarity solutions give $J_{0}=2.2$, and the integral $\sim 0.32$. Here we take $B=4, L=2$. The force components in the $x^{\prime}$ - and $y^{\prime}$-directions are

$$
\begin{aligned}
& F_{y^{\prime}}=F_{n} \cos \alpha+m_{21}^{\prime} \frac{\mathrm{d} U}{\mathrm{~d} t}, \\
& F_{x^{\prime}}=F_{n} \sin \alpha+m_{11}^{\prime} \frac{\mathrm{d} U}{\mathrm{~d} t},
\end{aligned}
$$

For a translating ellipse of major and minor axes $a$ and $b, m_{i j}$ are given by

$$
\begin{aligned}
& m_{11}=-\rho \pi b^{2}, \\
& m_{22}=-\rho \pi a^{2}, \\
& m_{12}=m_{21}=0 .
\end{aligned}
$$

The flat plate corresponds to $b=0, a=L / 2$. 


\subsection{Navier-Stokes simulations}

To obtain the unsteady force on an accelerating, non-rotating ellipse, we solve the two-dimensional Navier-Stokes equation in vorticity-stream function formulation in elliptic coordinates (Wang et al. 2004),

$$
\begin{aligned}
\frac{\partial(S \omega)}{\partial t}+(\sqrt{S} \boldsymbol{u} \cdot \nabla) \omega & =v \Delta \omega, \\
\nabla \cdot(\sqrt{S} \boldsymbol{u}) & =0 .
\end{aligned}
$$

where $\boldsymbol{u}$ is the velocity field, $\omega$ the vorticity field, and $S$ the local scaling factor $S(\mu, \theta)=C_{0}^{2}\left(\cosh ^{2} \mu-\cos ^{2} \theta\right)$ resulting from coordinates transformation. The derivatives are with respect to the elliptic coordinates $(\mu, \theta)$. The conformal transformation is given by

$$
x+\mathrm{i} y=c_{0} \cosh (\mu+\mathrm{i} \theta) .
$$

In the two-dimensional vorticity-stream function formulation, the non-inertial frame introduces only one extra term, the rotational acceleration of the wing, which is absent for the present case of strictly linear acceleration. Other non-inertial terms can be expressed as a gradient of a potential function. Thus they can be absorbed into the pressure term. The curl of the gradient of pressure is zero. The body motion is reflected in the far-field boundary conditions, and the no-slip boundary condition at the wing is enforced explicitly through the vorticity and stream function boundary conditions.

The velocity and vorticity are solved in the accelerating body coordinates, $(x, y)$, and are then transformed into the inertial frame. Navier-Stokes computations were performed for an ellipse with a constant acceleration along the negative $x^{\prime}$-axis (see figure 1), with $m=1, U(t)=4 t$, so $B=4$ in (2.1). In computational units, $a=L / 2=1$, $b=0.125$. The ellipse travels a chord length over a unit time in this case. The viscosity is chosen such that the $\operatorname{Re} \equiv U(t) L / v=800$ at $t=1$.

Figure 2 shows the vorticity field for the case of $\alpha=50^{\circ}$ at $t=0.2,0.4,0.6$, and 0.8 . The vortex centre locations calculated from (3.17) and (3.19), with $\omega_{0}(1) \approx-0.142+$ $0.320 \mathrm{i}$ from Pullin (1978) $(m=1)$ and $\omega_{1}(1)=\omega_{1 v}$ from equation (3.27), are plotted as a comparison. The inviscid model gives a rough approximation to the position of the center of vorticity for the separated flow near both the windward and leeward wing tips, with differences to the Navier-Stokes prediction attributable to both viscous and wing thickness effects. This will nevertheless be shown to provide a reasonable estimate of the unsteady vortex force.

\subsection{Forces on the ellipse}

The forces on the ellipse are calculated in the inertial frame by integrating the viscous stress

$$
\begin{aligned}
\boldsymbol{F}_{p} & =\rho v \int \frac{\partial \omega}{\partial r}(y,-x) \mathrm{d} s+\rho A_{w} \frac{\mathrm{d} U}{\mathrm{~d} t}, \\
\boldsymbol{F}_{v} & =\rho v \int \omega \hat{s} \mathrm{~d} s,
\end{aligned}
$$

where $\boldsymbol{F}_{p}$ and $\boldsymbol{F}_{v}$ denote pressure and viscous forces, $\rho$ is the fluid density, $A_{w}$ the total area of the wing and $\hat{s}$ the tangent vector. The second term in $\boldsymbol{F}_{p}$ is similar to the buoyancy force associated with hydrostatic pressure, i.e. fluids accelerate with $\mathrm{d} U / \mathrm{d} t$. In the special case of $\alpha=90^{\circ}$, the leading- and trailing-edge vortices have equal contributions, and the net force is in the $x$-direction. Wagner's theory is inapplicable 
(a)

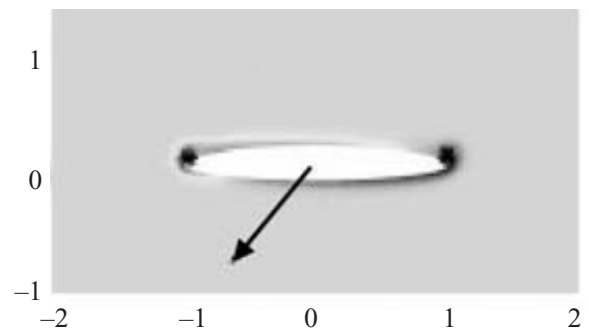

(c)

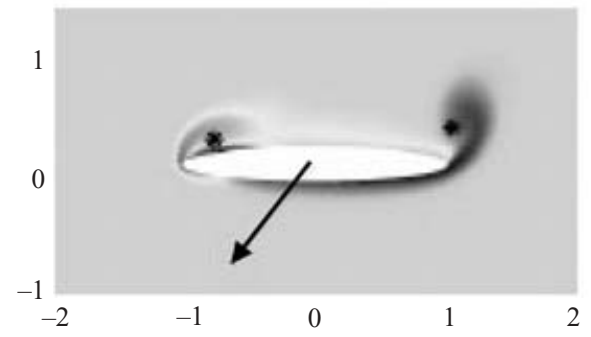

(b)

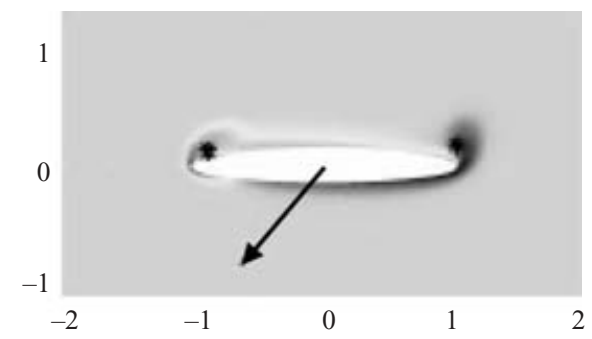

(d)

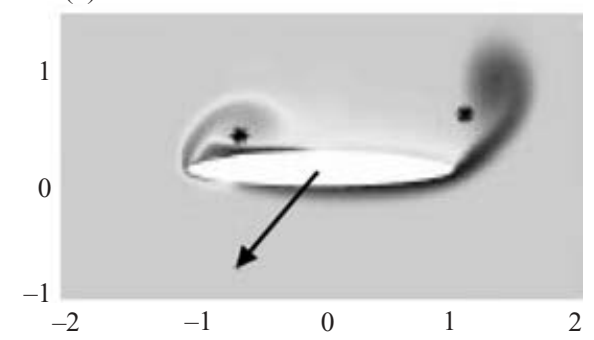

Figure 2. The vorticity field in the case of $\alpha=50^{\circ}$. Grey scale indicates the vorticity strength. The * mark the positions of the vortices calculated from the inviscid point-vortex model. The arrow indicates the direction of the acceleration. Axes labels are omitted. $(a) t=0.2,(b) 0.4$, (c) $0.6,(d) 0.8$. Other parameters are $m=1, U=4 t, L=2, e=0.125$.

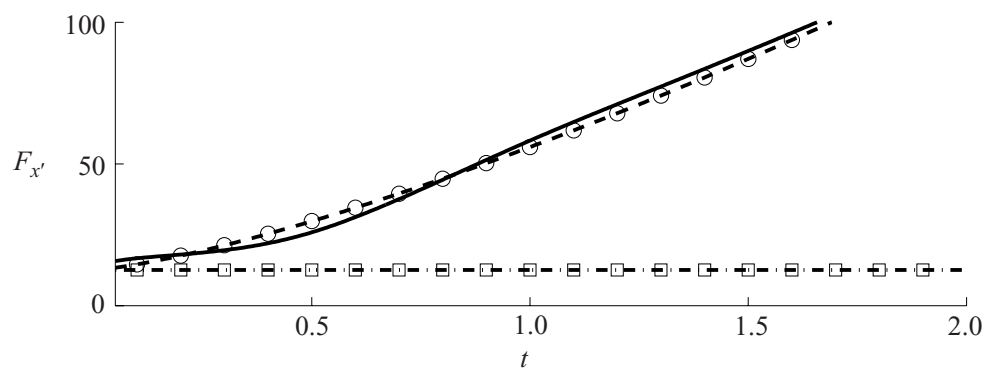

FIGURE 3. Comparison of theoretical and computational forces for a special case, $\alpha=90^{\circ}$ : present theory (dashed lines with circles) and computational results (solid line). Wagner's theory (dash-dotted line with squares) is inapplicable here. Ellipse thickness ratio, $e=0.125$, and the velocity, $U(t)=4 t$.

here, but the present theory is expected to work best in this case since the symmetry between the leading and trailing edge vortices is exact. It is seen in figure 3 that the theoretical prediction fits the computational force up to $t=2$, or equivalently, up to 4 chords of travel, beyond the small time scale assumed by the theory.

To be consistent with the conventional definition of lift and drag in airfoil theory, the forces are decomposed into drag, $F_{x^{\prime}}$, anti-parallel to wing motion, and lift, $F_{y^{\prime}}$ orthogonal to wing motion, indicated in figure 1. Figure 4 shows the comparison of the two theories and the computed force at angles seen in insect flight, $30^{\circ}-60^{\circ}$. As expected, Wagner's theory is a better approximation of lift $\left(F_{y^{\prime}}\right)$ at small angle of attack while the present theory works better at higher angle of attack. At $\alpha=30^{\circ}$, Wagner's theory follows the computed $F_{y^{\prime}}$ closely up to $t \sim 1.8$, while the present theory starts to deviate at $t \sim 1$. At $\alpha=60^{\circ}$, Wagner's theory overpredicts computed 

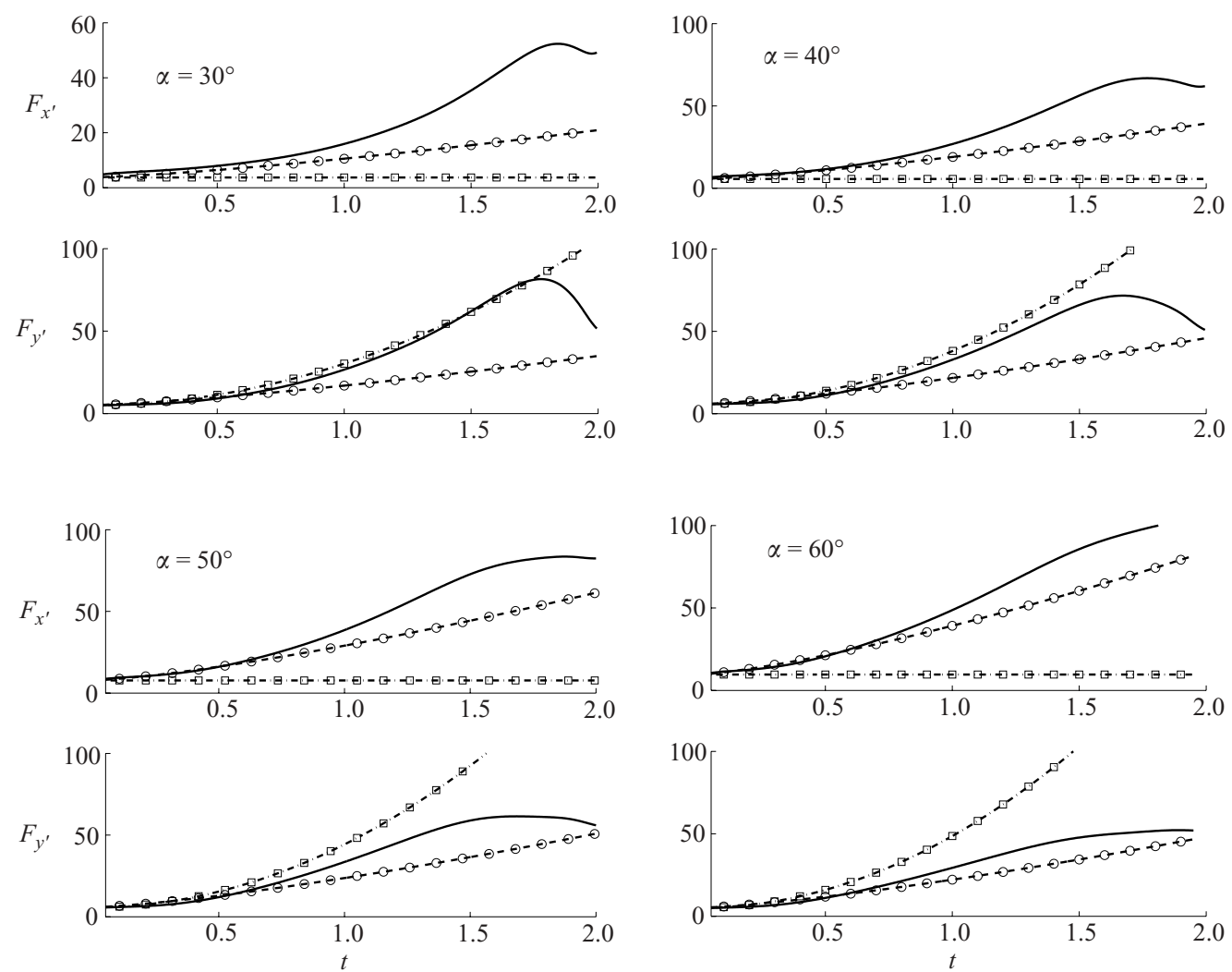

FIGURE 4. Comparison of theoretical and computational forces: present theory (dashed lines with circles); Wagner's theory (dash-dotted line with squares); and computational results (solid line). $e=0.125$, and $U=4 t$.

$F_{y^{\prime}}$ starting at $t \sim 0.5$, while the vortex theory agrees well up to $t \sim 2$. By definition Wagner's theory does not predict $F_{x^{\prime}}$ other than the component contributed by the attached-flow added mass. In contrast, the vortex theory is uniformly valid in $\alpha$ and so provides estimates of $F_{x^{\prime}}$ that appear to work especially well at stalled angles. NavierStokes calculations were also carried out for an ellipse of higher thickness ratio, $e=0.25$. The forces were found to be almost identical to the results for $e=0.125$, shown in figures 3 and 4.

\section{Application of vortex theory to insect hovering flight}

\subsection{Lift coefficients in single-stroke horizontal motion}

We now use the vortex theory to calculate the average normal forces produced by starting flow to time $t=t_{s}$, and use these to estimate unsteady aerodynamic forces in insect hovering flight. In $(x, y)$-coordinates, where $y$ is normal to the plate surface, the average normal forces on the plate to time $t=t_{s}$ are

$$
\overline{F_{y}^{(a)}}=\frac{1}{t_{s}} \int_{0}^{t_{s}} F_{y}^{(a)}(t) \mathrm{d} t, \quad \overline{F_{y}^{(v)}}=\frac{1}{t_{s}} \int_{0}^{t_{s}} F_{y}^{(v)}(t) \mathrm{d} t,
$$


where $\overline{F_{y}^{(a)}}, \overline{F_{y}^{(v)}}$ are the average added mass (attached flow) and vortex normal forces per unit length respectively. From (2.10) and (3.30), these are

$$
\begin{aligned}
& \overline{F_{y}^{(a)}}=\frac{\pi}{4} \rho L^{2} B \sin \alpha t_{s}^{m-1}, \\
& \overline{F_{y}^{(v)}}=2 \rho K J_{0} B^{5 / 3} L^{4 / 3} \sin ^{5 / 3} \alpha t_{s}^{5(1+m) / 3-2} \operatorname{Re}\left[\int_{0}^{1} \omega_{0}^{1 / 2}(\lambda) \mathrm{d} \lambda\right],
\end{aligned}
$$

where (3.4) has been used.

We define two normal force coefficients for power-law motion for time $t_{s}$ by

$$
C_{y}^{(a)}=\frac{\overline{F_{y}^{(a)}}}{\frac{1}{2} \rho L \bar{u}^{2}}, \quad C_{y}^{(v)}=\frac{\overline{F_{y}^{(v)}}}{\frac{1}{2} \rho L \bar{u}^{2}},
$$

where $\bar{u}=S / t_{s}$ is the mean speed and the stroke length is

$$
S=\frac{1}{1+m} B t_{s}^{1+m} .
$$

In order to make comparisons with measurements and computation for a single wing stroke in the horizontal direction at angle of attack $\alpha$, it is convenient to rotate to $\left(x^{\prime}, y^{\prime}\right)$-axes, with corresponding lift coefficients

$$
C_{L}^{(a)}=C_{y}^{(a)} \cos \alpha, \quad C_{L}^{(v)}=C_{y}^{(v)} \cos \alpha, \quad C_{L}=C_{L}^{(a)}+C_{L}^{(v)} .
$$

These can now be estimated by use of (5.2) and (5.3). When (5.5) is used to eliminate $B$, it is found that

$$
\begin{aligned}
& C_{L}^{(a)}=\frac{\pi}{2}(1+m) \sin \alpha \cos \alpha\left(\frac{L}{S}\right), \\
& C_{L}^{(v)}=4 J_{0}(1+m)\left(\frac{3}{4}\right)^{2 / 3}\left(\frac{L}{S}\right)^{1 / 3} \sin ^{5 / 3} \alpha \cos \alpha \operatorname{Re}\left[\int_{0}^{1} \omega_{0}^{1 / 2}(\lambda) \mathrm{d} \lambda\right] .
\end{aligned}
$$

Of interest is the $(L / S)^{1 / 3}$ dependence of the vortex-lift coefficient with exponent independent of $m$. To obtain numerical estimates, the wing chord is taken as $L=0.01 \mathrm{~m}$ with stroke $S=0.025 \mathrm{~m}$ and frequency $f=40 \mathrm{~Hz}$ giving $t_{s}=0.0125 \mathrm{~s}$. These are typical of dragonfly wings. The lift coefficients for $m=0.5$ are shown in figure 5. It can be seen that the lift is dominated by the vortex force but the attached-flow contribution to the single-stroke lift coefficient is not negligible. From (5.8) the vortex lift is maximum at $\alpha=\operatorname{arcos}(\sqrt{3 / 8}) \approx 52.2^{\circ}$, independent of $m$ while the attached-flow single-stroke lift is maximum at $\alpha=45^{\circ}$. The total lift coefficient is maximized at a value of $\alpha$ somewhere between these values. Thus the vortex-lift theory provides a mechanism for delayed/dynamic stall.

Total lift coefficients for three values of $m$ are shown in figure 6 compared to the results of experiment (Dickenson \& Götz 1993) and direct simulation (Wang $2000 a$ ), at Reynolds number of order 200. Both the experiments and the numerics were obtained for a wing moving at constant velocity at a fixed incidence. Therefore, the comparison here is mainly about the $\alpha$ dependence of $C_{L}$.

\subsection{Forces in hovering flight}

We now make numerical estimates of time-averaged forces in hovering flight for two drastically simplified profiles of wing motion. In each case the wing is modelled in two-dimensional flow by the present flat plate of chord $L$ which moves in a periodic 


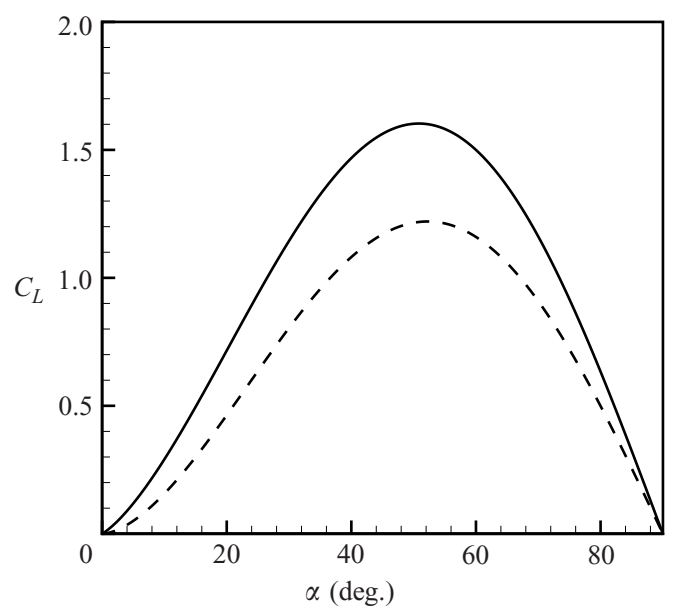

FigURE 5. Lift coefficients, $m=1 / 2 . L=0.01 \mathrm{~m}, S=0.025 \mathrm{~m}$. Dashed line, vortex-lift coefficient $C_{L}^{(v)}$. Solid line, total-lift coefficient $C_{L}$. The difference is the attached-flow lift coefficient $C_{L}^{(a)}$.

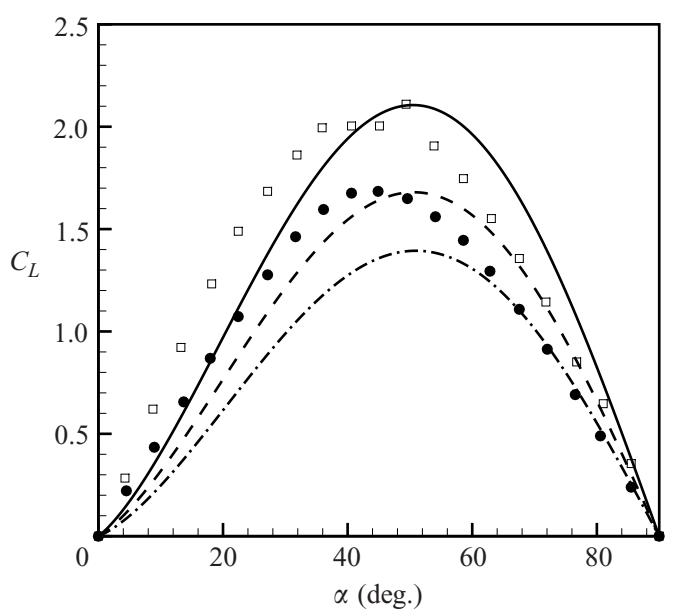

Figure 6. Total lift coefficients $C_{L}$ during lift stroke. Dash-dot line, $m=0$; dashed line, $m=1 / 2$; solid line, $m=1$. $L=0.01 \mathrm{~m}, S=0.025 \mathrm{~m}$. Filled circles, direct numerical simulation, $R e=192$ (Wang 2000a); open squares, experiment (Dickenson \& Götz 1993).

motion with frequency $f=1 / T$ where $T=2 t_{s}$ is the period. The first profile, denoted $\mathrm{I}$, is an approximation to that used in the numerical simulations of Wang (2000b), where the wing moves in a combined pitching and heaving motion along a line at $\pi / 3$ to the horizontal. For simplicity we ignore the pitching motion, so that the wing remains horizontal (parallel to the $x$-axis) during a downward, or lift stroke, and moves a stroke length $S$ in the time interval $0 \leqslant t \leqslant t_{s}$ as described in $\S 2.1$, at a constant angle $\alpha=\pi / 4$ to the negative $x$-axis. It is this stroke that produces the entire vortex force during the wing cycle. During the interval $t_{s} \leqslant t \leqslant T$, the wing sheds the vortices by a return motion unspecified here but similar to that described by Wang $(2000 b)$. During the return stroke, the wing produces no overall contribution to the force save that required to just cancel the attached-flow force (2.10); it is well-known that this force must average zero in the periodic translational motion of a rigid 


$\begin{array}{ccccc}m & F_{L}\left[\mathrm{~N} \mathrm{~m}^{-1}\right] & \Gamma_{+}\left(t_{s}\right)\left[\mathrm{m}^{2} \mathrm{~s}^{-1}\right] & \Gamma_{-}\left(t_{s}\right)\left[\mathrm{m}^{2} \mathrm{~s}^{-1}\right] & \delta / L \\ 0 & 0.018 & 0.019 & -0.066 & 0.51 \\ 1 / 2 & 0.021 & 0.028 & -0.088 & 0.51 \\ 1 & 0.025 & 0.037 & -0.11 & 0.42\end{array}$

TABLE 1. Average force and other parameters, wing cycle I. $L=0.01 \mathrm{~m}$, $S=0.025 \mathrm{~m}, t_{s}=0.0125 \mathrm{~s}$.

$\begin{array}{ccccc}m & F_{L}\left[\mathrm{~N} \mathrm{~m}^{-1}\right] & \Gamma_{+}\left(t_{s}\right)\left[\mathrm{m}^{2} \mathrm{~s}^{-1}\right] & \Gamma_{-}\left(t_{s}\right)\left[\mathrm{m}^{2} \mathrm{~s}^{-1}\right] & \delta / L \\ 0 & 0.027 & 0.028 & -0.071 & 0.55 \\ 1 / 2 & 0.030 & 0.039 & -0.095 & 0.49 \\ 1 & 0.037 & 0.051 & -0.121 & 0.45\end{array}$

TABLE 2. Average force and other parameters, wing cycle II. $L=0.01 \mathrm{~m}$, $S=0.025 \mathrm{~m}, t_{s}=0.0125 \mathrm{~s}$.

two-dimensional body. With this model of the wing motion, the average vortex force over the period $T$ in the $y$-direction, taken as the vertical for this cycle, is then one-half of that given by (5.3). The average force per unit length averaged over $T=2 t_{s}$ can now be expressed as

$$
\begin{aligned}
F_{y} & =\frac{1}{2} \overline{F_{y}^{(v)}} \\
& =\frac{1}{4} \rho L\left(\frac{S}{t_{s}}\right)^{2} C_{y}^{(v)} .
\end{aligned}
$$

In this expression, only the vortex force contributes. We utilize previous values for $L, S$ and $t_{s}$, with $\alpha=\pi / 4$. Values for the average normal force and other parameters defined previously for three values of $m$ are shown in table 1. Values of the vortex force per unit span are somewhat lower than that found by Wang (2000b), $0.026 \mathrm{~N} \mathrm{~m}^{-1}$, from unsteady simulations of sinusoidal motion at Reynolds number of 1256 based on chord and maximum velocity. The normal force shows rather weak dependence on $m$ when $S$ is fixed. Also shown in table 1 are point-vortex estimates of the circulations shed from the two wing edges at $t=t_{s}$, and $\delta\left(t_{s}\right) / L$. It can be seen that this ratio not small compared with unity as formally required by the theory but the estimated size of the shed vortices is nevertheless of order those found by Wang (2000b) towards the end of the thrust stroke.

In a second model wing cycle, denoted II, it is convenient to work in $\left(x^{\prime}, y^{\prime}\right)$-axes, where the plate centerline moves with power-law velocity along the negative $x^{\prime}$-axis in $0 \leqslant t \leqslant t_{s}$, remaining at constant angle of attack $\alpha=52.2^{\circ}$. The lift coefficient is then given by (5.8). At $t=t_{s}$, the wing halts and rotates instantaneously through $75.6^{\circ}$ clockwise, shedding the vortex pair, and then executes a power-law motion in the positive $x^{\prime}$-direction at angle of attack $\alpha=52.2^{\circ}$. Following further instantaneous deceleration and counterclockwise rotation, the cycle is repeated. If it is assumed that the half-cycle rotations do not contribute to the overall lift (this could be estimated using an extension of the present model; see also discussion in $\S 6$ ), the time-averaged vortex force in the $y^{\prime}$-direction, taken as the vertical for this cycle, can be obtained from (5.8). Numerical values shown in table 2 are substantially larger than those obtained for cycle I. 


\section{Discussion}

The present theory provides a theoretical basis for two effects observed in studies of insect flight. First, it is well-known that most hovering insects employ angles of attack much higher than the stalled angle of an airfoil. Typical values during the translational phase are about $25^{\circ}-45^{\circ}$ in normal hovering (Ellington 1984). Dragonflies and butterflies employ even higher angles of attack. At these 'stalled' angles, the wing can generate higher transient lift coefficients compared to the steady-state value, a phenomenon called dynamic stall. Recent discussions have mainly focused on the role of dynamic stall on lift enhancement (Dickinson \& Götz 1993; Ellington et al. 1996; Wang 2000b). A side effect of dynamic stall is the increase of drag. In fact at such high angle of attack, it is no longer most convenient to separate lift and drag in the traditional sense, which was appropriate for an unstalled airfoil. Wang recently argued that insects might use both lift and drag to manoeuvre in air (Wang 2003). In particular, a wing executing idealized kinematics similar to those used by dragonflies uses mostly pressure drag to generate the vertical force to hover (Wang 2003). Classical steady and unsteady airfoil theories, however, were designed to treat the regime of small angle of attack where the flow is attached at the leading edge; they do not predict pressure drag. To extend these theories to a full range of angle of attack, it is necessary to include both the leading- and trailing-edge vortices. The theory presented here is a second-order unsteady theory for a stalled airfoil. In $\S 5.1$ it was shown that the combination of vortex and attached-flow added mass forces produces a maximum lift coefficient at an angle between $45^{\circ}$ and $52.2^{\circ}$, in fair agreement with observation.

Second, a reciprocating insect wing must accelerate and decelerate periodically near wing reversal. The unsteady forces associated with such an acceleration are neglected in the classical quasi-steady theory, which includes only the term depending on the translational velocity (Weis-Fogh \& Jensen 1956; Weis-Fogh 1973). Added mass (attached flow) is the simplest contribution to the force due to wing acceleration, but is not necessarily the dominant contribution. Another effect of the wing acceleration on the force generation is related to the unsteady growth of vorticity and the presence of the vorticity near to the wing. Their effects have been investigated in the recent experiments and computations of two families of wing kinematics. One consists of constant translation in the middle of the stroke and sharp accelerations near the end of the stroke (Dickinson et al. 1999; Sun \& Tang 2002), and another simple sinusoidal motions (Wang, Birch \& Dickinson 2004). The differences between the measured forces and the steady-state theory based on translational velocity alone have been a subject of recent discussions. The proposed mechanisms for the observed peaks near wing reversal include the wing-wake interaction (Dickinson et al. 1999; Birch \& Dickinson 2003), wing rotation during reversal (Dickinson et al. 1999; Sane $\&$ Dickinson 2002), and wing acceleration (Sun \& Tang 2002). All of them still require further clarification and theoretical analysis.

The present analysis allows quantification of unsteady forces due to acceleration near the wing reversal. For power-law start-up velocity given by (2.1), the contribution of pure added mass and separated vorticity provides start-up forces proportional to $t^{m-1}, m \neq 0$, and $t^{(5 m-1) / 3}$ respectively. If wing reversal is modelled by impulsive start-up, $m=0$, at $t=0$, the corresponding forces are proportional to $\delta(t)$, where here $\delta$ denotes the Dirac delta function, and $t^{-1 / 3}$ respectively, and are therefore unbounded when $t \rightarrow 0^{+}$. This suggests peaked starting forces in periods of rapid acceleration/deceleration at wing reversal. Figure 7 shows an example of the force coefficient as a function of time for $m=1$. Note that the initial transient coefficients 

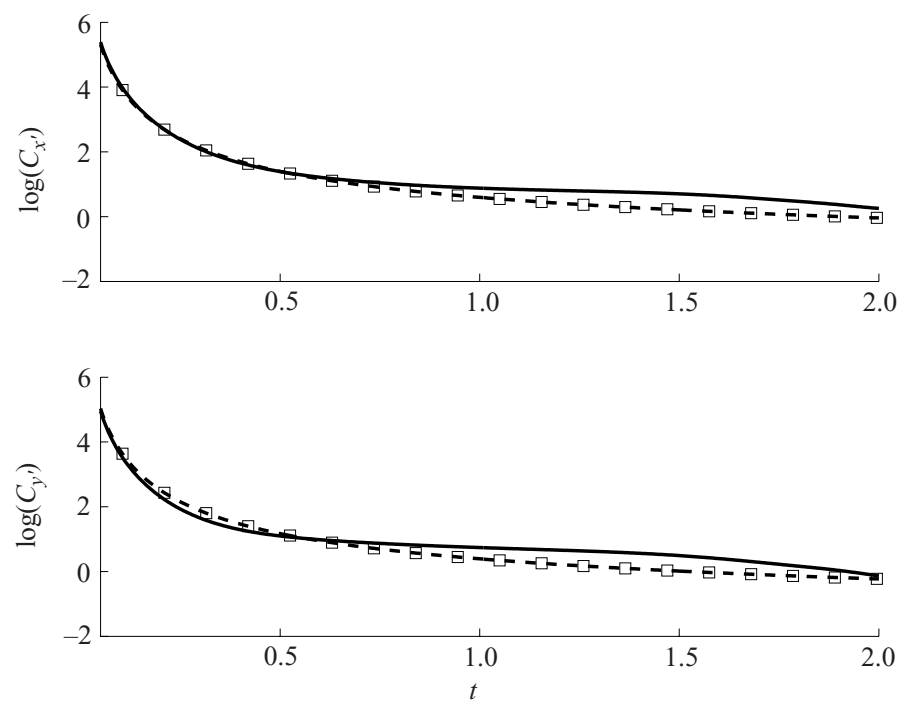

FIGURE 7. Dimensionless force coefficients for $\alpha=50^{\circ}$, normalized by $\frac{1}{2} \rho U^{2}(t) L$ : present theory (dashed lines with squares) and computational results (solid line).

are much higher than the steady-state value. This is associated with the fact that the wing acquires an instantaneous non-zero force at $t=0^{+}$where $U(t)=0^{+}$. This surprising result was already predicted by Wagner's theory that an impulsively started wing instantaneously acquires half of its final lift. In the present context, this nonzero force at zero velocity, or extremely high lift coefficients, may explain in part the force peaks near wing reversal as seen in experiments and computations of reciprocal motions (Dickinson et al. 1999; Sun \& Tang 2002; Wang et al. 2004). It is not clear, however, that this is advantageous to insect flight: the three-dimensional simulations of Sun \& Tang (2002) show both positive and negative spikes in lift coefficient, relative to the mean-cycle value, during wing reversal, with expected cancellations. This is consistent with earlier assumptions in our modeling of wing cycle II.

\section{Concluding remarks}

In obtaining the main analytical result of this paper, the unsteady vortex force given by (3.31), we have invoked several simplifications. The strongest may be the assumption of two-dimensional flow. Yet even for a three-dimensional lifting surface in accelerated motion, the initial, near-edge vortex generation process is expected to be approximately two dimensional for short times. Even given the two-dimensional model, our description of the growth of the edge vortices further neglects the effects of finite viscosity and its effect in producing secondary separation of the wing boundary layer induced near the wing edge by the unfavourable pressure gradient produced by the primary separated vortex; see figure 2 at $t=0.8$. This is known to modify the near-edge pressure distribution. Despite these gross omissions the good agreement obtained between forces predicted by the vortex model, and fully resolved NavierStokes simulation at moderate Reynolds number indicates that the former captures approximately the dynamics of the dominant vortex growth mechanism in the local lift generation process on the accelerating wing. The second part of the lift-production mechanism, in which the insect produces a directed train of vortices as the agent of 
momentum flux, requires further study. In two dimensions, the model of the insect sitting atop a column, spray, or sea of vortex dipoles (Wang 2000a) seems physically appropriate. In three dimensions the means by which the insect organizes coherent vortex structures remains an area for future research. Complex vortex dynamics can be anticipated, which may have an impact on issues of stability and control.

D. I. P. wishes to thank P. E. Dimotakis for helpful discussions. Z. J.W. wishes to acknowledge the support by NSF Early Career grant, ONR YIP, AFOSR and the Packard Foundation.

\section{REFERENCES}

Birch, J. M. \& Dickinson, M. H. 2003 The influence of wing-wake interactions on the production of aerodynamic forces in flapping flight. J. Expl Biol. 206, 2257-2272.

Childress, S. 1981 Swimming and Flying in Nature. Cambridge University Press.

Cortelezzi, L. \& Leonard, A. 1993 Point-vortex model of the unsteady separated flow past a semi-infinite plate with transverse motion. Fluid Dyn. Res. 11, 263-295.

Dickinson, M. H. 1996 Unsteady mechanisms of force generations in aquatic and aerial locomotion. Am. Zool. 36(6), 537-554.

Dickinson, M. H. \& GöTZ, E. K. 1993 Unsteady aerodynamic performance of model wings at low Reynolds numbers. J. Expl Biol. 174, 45.

Dickinson, M. H., Lehmann, F. O. \& Sane, S. P. 1999 Wing rotation and the aerodynamic basis of insect flight. Science 284, 1954-1960.

Ellington, C. P. 1984 The aerodynamics of hovering insect flight i.-v. Phil. Trans. R. Soc. Lond. B 305, $1-181$.

Ellington, C. P., Van Den Berg, C., Willmott, A. P. \& Thomas, A. L. R. 1996 Leading edge vortices in insect flight. Nature 384, 626.

Freymuth, P., Gustafson, K. \& Leben, R. 1991 Visualization and computation of hovering mode. In Votex method and vortex motion (ed. K. Gustavson \& J. Sethian), p. 143. Philadelphia: SIAM.

Graham, J. M. R. 1983 The lift on an airfoil in starting flow. J. Fluid Mech. 133, 413-425.

JONES, M. 2003 The separated flow of an inviscid fluid around a moving flat plate and the unsteady kutta condition. J. Fluid Mech. 496, 405-441.

von Kármán, T. \& Burgers, J. M. 1963 General Aerodynamic Theory - Perfect Fluids. Springer.

von Kármán, T. \& Sears, W. R. 1938 Airfoil theory for non-uniform motion. J. Aero. Sci. 5(10), 379-390.

Lighthill, M. J. 1977 Introduction to the scaling of aeriel locomotion. In Scaling Effects in Animal Locomotion (ed. T. Pedley) p. 365. Academic.

Maxworthy, T. 1981 The fluid dynamics of insect flight. Annu. Rev. Fluid Mech. 13, 329.

Milne-Thomson, L. M. 1968 Theoretical Hydrodymanics, 5th edn. MacMillan.

Newman, J. N. 1977 Marine Hydrodymanics, 1st edn. The MIT Press.

PULlin, D. I. 1978 The large-scale structure of unsteady self-similar rolled-up vortex sheets. J. Fluid Mech. 88, 401-430.

RAYNeR, J. 1979a A vortex theory of animal flight. Part 1. The vortex wake of a hovering animal. J. Fluid Mech. 91, 697.

RAYNER, J. $1979 b$ A vortex theory of animal flight. Part 2. The forward flight of birds. J. Fluid Mech. 91, 731.

RotT, N. 1956 Diffraction of a weak shock with vortex generation. J. Fluid Mech. 1, 111.

Saffman, P. G. 1992 Vortex Dynamics, 1st edn. Cambridge University Press.

SANE, S. \& Dickinson, M. H. 2002 The aerodynamic effects of wing rotation and a revised quasi-steady model of flapping flight. J. Expl Biol. 205, 1087-1096.

Smith, J. 1966 Theoretical work on the formation of vortex sheets. Prog. Aero. Sci. 7, 35-51.

SUN, M. \& TANG, J. 2002 Lift and power requirements of hovering flight in drosophilia virilis. J. Expl Biol. 205, 2413-2427. 
WaGNER, H. 1925 Über die entstehung des dynamischen auftriebes von tragflüeln. Z. Angew. Math. Mech. 5, 17-35.

Walker, P. B. 1931 Experiments on the growth of circulation about a wing. Tech. Rep. Aero. Res. Committee No. 1402.

Wang, Z. J. 2000a Two-dimensional mechanism for insect hovering. Phys. Rev. Lett. 85, 2216-2219.

WANG, Z. J. $2000 b$ Vortex shedding and frequency selection in flapping flight. J. Fluid Mech. 410, 323-341.

WANG, Z. J. 2003 Using drag to hover. J. Expl Biol. (submitted); http://arxiv.org/ps/physics/ 0304069 .

WANG, Z. J., BIRCH, J. \& Dickinson, M. H. 2004 Unsteady forces in hovering flight: Computation vs experiments. J. Expl Biol. 207, 447.

WEIS-FogH, T. 1973 quick estimates of flight fitness in hovering animals, including novel mechanisms for lift production. J. Expl Biol. 59, 169-230.

Weis-Fogh, T. \& Jensen, M. 1956 Biology and physics of locust flight. Proc. R. Soc. B 239 239, 415-584. 